THE METAL-RICH RR LYRAES: THEIR ORIGIN AND BEARING ON THE AGE AND HELIUM ABUNDANCE OF THE GALACTIC DISK

Robert P. Kraft

Lick Observatory, Board of Studies in Astronomy and Astrophysics University of California, Santa Cruz 95064

\title{
Abstract
}

We consider further the question of the origin and evolution of metal-rich RR Lyraes (MR-RR's) in the solar vicinity, continuing the discussion begun by Taam, et al (1976) who showed that MR-RR's descend from red giants of Eggen's old disk population. If an old disk giant (ODG) is similar to a giant in a galactic cluster like NGC 188, then it must lose $0.5{ }^{2 l}{ }_{\theta}$ if it is to pump the MR-RR domain; thus the probability that an ODG will metamorphose into an RR Lyrae is about $1 / 200^{\text {th }}$ the probability that its metal-poor (halo) counterpart will do so, else the sky would be full of bright MR-RR's.

The MR-RR's of the solar vicinity are unique in having $\left\langle\mathrm{P}_{\mathrm{ab}}\right\rangle$ for the Bailey ab's shorter than that of Oosterhoff Type I RR Lyraes; the former have $\left\langle P_{a b}\right\rangle=0.45$ (Preston 1959), whereas the 1atter have $\left\langle P_{a b}\right\rangle=0.55$ as, for example, in M 3 and M 5. According to the recent work of McDonald (1976), $\left\langle M_{v}\right\rangle(M R-R R ' s) \stackrel{\sim}{=}+0.80$, whereas Type I and Type II RR-Lyraes of the halo have $\left\langle M_{v}\right\rangle=+0.55$. There exists one globular cluster, viz., NGC 6712, having metalrich RR Lyraes, yet these have $\left\langle P_{a b}\right\rangle=0.56$, as in a Type I cluster. 
We show that the lower luminosity and shorter period of field MR-RR's is compatible with the view that there is a stellar component of the ancient galactic disk population having helium abundance $Y$ about $0.07 \pm 0.03$ smaller than that of globular clusters.

This result is a simple extrapolation of the recent seminal discussion (Demarque and MCClure 1977) of the probable difference in $Y$ of NGC 2420, a moderately old galactic (disk) cluster, and 47 Tuc, a metal-rich globular cluster (1ike NGC 6712), and supports the earlier result of Gross (1973). Consequences of the assumption of low disk helium on ODG mass-loss rates, following the work of Fusi-Pecci and Renzini (1975), are explored. It is likely that the metal-rich cluster NGC 6712 must be essentially as old as the halo clusters if it is to produce RR Lyraes at all, and the old disk must be a little younger.

Explored also is the consequence of low disk helium on the observed properties of ODG's, plenetary nebulae, and old disk dwarfs (ODD's). If these have $Y$ roughly correlated with $Z$ with decreasing age such that $\frac{\Delta Y}{\Delta Z} \sim 4$, then $L\left(t_{9}\right)$ of $0 D D^{\prime}$ 's will be constant with time, as evidently required by observation (Eggen 1973b). 


\section{Introduction: Statement of the Problem}

We consider here the origin and evolution of metal-rich RR Lyraes (MR-RR's), a group comprising about $25 \%$ of the RR Lyrae star population in the vicinity of the sun. By "metal-rich", we mean the RR Lyraes having a Preston (1959) index $\Delta s \leq 2$ and $\langle\Delta s\rangle=+0.6$, so that $[\mathrm{Fe} / \mathrm{H}] ;-0.6$ or $\langle[\mathrm{Fe} / \mathrm{H}]\rangle=-0.3$, according to the calibration of Butler (1975a). Thus for MR-RR's the degree of "metal richness" depends on one's point of view: they are mildly metal-poor with respect to the Hyades and the sun, but metal-rich when conipared to RR Lyraes found in halo globular clusters.

In a recent paper, Taam, Kraft, and Suntzeff (1976) concluded that MR-RR's descend from red giants of Eggen's (1973a ) old disk population, a group having similar metal-abundance and galactic kinematics. 01d disk giants (ODG's) are generally assumed to be like the giants found in the oldest galactic clusters, e.g. NGC 188 and $M 67$, in which stars turning off the main sequence have masses near $1.0 \mathrm{~m}_{\theta}$ (cf. Sandage and Eggen 1969). According to the scaled solar-wind mass-loss theory of Fusi-Pecci and Renzini (1975), these stars shed about $0.2 \mathbb{N}_{0}$ as they ascend the red-giant branch to the helium core flash. However, stellar evolutionary theory (SET) (cf., e.g., Taam, et al 1975, Sweigart and Gross 1976) constrains metal-rich stars of the horizontal branch $(H B)$ to have iff $0.5-0.6 \mathbb{M}_{\theta}$ if they are to occupy the instability strip. Taam, et al therefore concluded that only a small fraction of ODG's, i.e., those with larger-than-average mass loss, actually succeed in pumping the MR-RR domain. The problem of occupation, it should be noted, does not arise among the metal-poor RR Lyraes (MP-RR's) of the halo: compared to the MR-RR case, SET predicts that MP-RR's should have larger masses, viz., 
$\sim 0.72 l_{\theta}$ (Sweigart and Gross 1976), in accordance with fundamental precepts advanced long ago by Faulkner (1966). Moreover, the turnoff mass for halo globular clusters is in the range 0.8 to $0.9 \mathrm{~m}_{\theta}$ (Sandage 1970, Iben 1974), smaller than that for old disk galactic clusters such as NGC 188. Since both theory (Fusi-Pecci and Renzini 1975) and observation (Cohen 1975) suggest a pre-He core flash mass-loss of about $0.2 \mathfrak{m}_{0}$, halo (globular cluster) giants have little trouble becoming MP-RR's. Based on star counts, Taam et al concluded that ODG's have a probability of becoming RR Lyraes some 200 times smaller than that of their metal-poor counterparts, presumably because only a small fraction of the former lose enough mass to pump the MR-RR domain.

The suggestion of Taam, et al, although addressing the problem of the origin of these stars, sheds no light on several other anomalous aspects of the group. First among these is the virtual lack of representation of MR-RR's in metal-rich globular clusters. Among the half-dozen well-studied metal-rich clusters of the disk and nuclear bulge, one finds 47 TuC, M 69, NGC 6356 and $M 71$ which contain only three RR Lyraes altogether, yet if they were metalpoor halo clusters, they would collectively contain a few hundred such objects. From a small sample of RR Lyraes in NGC 6171 (Sandage and Katem 1964 and references therein, Dickens 1970), Butler (1975a) found $\langle\Delta s\rangle=3.7 \pm 0.6$ (m.e.) corresponding to $\langle[\mathrm{Fe} / \mathrm{H}]\rangle=-0.8 \pm 0.1$ (m.e.); the cluster therefore lies just outside the range of interest. Only the 10 RR Lyraes of NGC 6712 (Sandage and Smith 1966), for which $\langle\Delta s\rangle=1.0 \pm 0.9$ (m.e.) (Butler 1975a), $\langle[\mathrm{Fe} / \mathrm{H}]\rangle=-0.4 \pm 0.1$ (m.e.), have $[\mathrm{Fe} / \mathrm{H}]$ in the required domain. But when the periods of these stars are compared with those of their field counterparts having the same metal abundance, a second curious anomaty emerges. 
Oosterhoff (1939) noted many years ago that globular clusters could be divided into two groups on the basis of the mean period $\left\langle\mathrm{P}_{a b}\right\rangle$ of the Bailey $a^{\prime}$ 's and b's. It was later shown (cf., e.g., Preston 1959, 1961, Kinman 1959, Arp 1965) that Type I clusters, for which $\left\langle P_{a b}\right\rangle=0.55$, are typically more metal-rich than Type II clusters, for which $\left\langle P_{a b}\right\rangle=0.65$. Crudely, Type I (e.g., M 5, M 3) and Type II (e.g., M 92, M 15) clusters have $[\mathrm{Fe} / \mathrm{H}] \ldots-1$ and -2 , respectively. Moreover, among the halo and disk RR Lyraes in the solar vicinity, Preston (1959) showed that there exists a broad correlation between $\left\langle\mathrm{P}_{a b}\right\rangle$ and $\Delta s$, therefore $[\mathrm{Fe} / \mathrm{H}]$. Of interest here is that the field metal-rich RR Lyraes have $\left\langle P_{a b}\right\rangle=0$. 45 , which is significantly smaller than $\left\langle\mathrm{P}_{\mathrm{ab}}\right\rangle$ for 0osterhoff Type I clusters, as one would expect since they have $\langle[\mathrm{Fe} / \mathrm{H}]\rangle=-0.3$. Yet in the metal-rich cluster NGC 6712, we find $\left\langle P_{a b}\right\rangle=0.56$ (Sandage, Smith and Norton 1966; Rosino 1966) as in a Type I cluster! of course, there are only 7 Bailey ab's in NGC 6712, but none has $P<0.50$, and the "standard deviation" of $\left\langle\mathrm{P}_{a b}>\right.$ is \pm 0.06 ; the period anomaly therefore seems significant. What meaning is to be attached to this? If we regard the MR-RR's of NGC 6712 as anomalous, then they are doubly mysterious: First, why do we find them in NGC 6712 but not in the other metal-rich clusters? And second, why do they have $\left\langle\mathrm{P}_{\mathrm{ab}}\right\rangle 20 \%$ longer than that of their (generously represented) field counterparts?

Valuable new information on temperatures and Tuminosities for MR-RR's has recently been provided by McDonald (1976) who, applying a modified BaadeWesselink method, obtained $\left\langle T_{e}\right\rangle$ and $\left\langle M_{v}\right\rangle$ as a function of $\Delta s$ for 47 solar neighborhood RR Lyraes of all metal abundances. Intrinsic colors of Bailey $a b$ ! $s$ were derived using $H_{\beta}$ line-strengths $W_{H_{B}}$ (modified Strömgren photometry), 
and the effect of metal abundance on the relationship between (B-V) and $W_{H_{B}}$ was determined from a grid of blanketed model atmospheres provided by

R. L. Kurucz. Using the models, values of $\mathrm{T}_{e}$ were also derived from the colors. These, along with $\langle(B-V)\rangle$ (at mean and minimum light) and $\left\langle M_{v}\right\rangle$, are given in Table 1.

Table 1.

Temperatures, Colors, and Absolute Magnitudes for Field RR Lyraes

of Bailey Type ab (after McDonald 1976)

\begin{tabular}{|c|c|c|c|c|c|c|c|}
\hline Type of RR Lyrae & $\begin{array}{l}\left\langle\mathrm{P}_{\mathrm{ab}}\right\rangle \\
\text { (days) }\end{array}$ & $\begin{array}{l}\left\langle\mathrm{P}_{t r^{*}}\right. \\
\text { (days) }\end{array}$ & $\begin{array}{l}\langle(B-V)\rangle \\
(\text { at mean } \\
\text { light) }\end{array}$ & $\begin{array}{l}\langle(B-V)\rangle \\
(\text { at min. } \\
\text { 1fght) }\end{array}$ & $\begin{array}{l}<\mathrm{T}_{\mathrm{e}}>\left({ }^{\circ} \mathrm{K}\right) \\
\text { (at mean } \\
1 \text { ight) }\end{array}$ & $\begin{array}{c}\left\langle M_{v}\right\rangle \\
\text { (at mean } \\
\text { light) }\end{array}$ & $\begin{array}{l}\text { No. } \\
\text { Stars }\end{array}$ \\
\hline Oosterhoff II & 0.65 & 0.550 & 0.35 & 0.44 & 6570 & +0.55 & 15 \\
\hline Oosterhoff I & 0.55 & 0.455 & 0.335 & 0.41 & 6790 & +0.58 & 19 \\
\hline MR-RR & 0.45 & 0.33 & 0.37 & 0.45 & 6825 & +0.82 & 13 \\
\hline
\end{tabular}

*For Types I and II, $P_{t r}$ from van Albada and Baker (1973); for MR-RR's, $P_{t r}$ estimated by the author, but see also Stepien (1972).

McDonald estimates that the overal1 internal accuracy in $\left\langle M_{v}\right\rangle$ is about \pm 0.20 (m.e.) in each group; thus the evidence that MR-RR's are somewhat fainter than the MP-RR's of the classical Oosterhoff groups is fairly firm. On the other hand, the RR Lyraes of the two 0osterhoff groups appear to have about the same brightness, although a small difference could not be ruled out, considering the size of the errors. A difference of about $0.1 \mathrm{mag}$ is expected (M 15 stars being the brighter) on the basis of a study of moderately metal-poor vs. very 
metal-poor RR Lyraes in w Cen (Butler, Be11, Dickens, and Epps 1978). By comparison, the Christy (1966) theory leads to differences in $\left\langle M_{v}\right\rangle$ considerably larger than those either of Table 1 or the $w$ Cen result: as $P_{t r}$ decreases, we compute $\left\langle M_{v}\right\rangle=+0.62,+0.97,+1.55$ for the three groups. The applicability of the theory, however, has been criticized on other grounds (van Albada and Baker 1973, Stellingwerf 1975), and the situation is unclear. Statistical parallaxes (Woolley, et al 1965) also have led to the conclusion that the luminosities of RR Lyraes increase with $\Delta s$, but these results were biased by the inclusion of stars now assigned to the AI Vel (RRS) group, and more recent studies (Hemenway 1975) neither support nor rule out the expected correlation. However, in studies based on stellar motions, any possible variation of $\left\langle M_{v}\right\rangle$ with $\Delta S$ is essentially masked by the large errors $( \pm 0.5$ mag) introduced when the sample is divided into small groups in intervals of $\Delta s$. In what follows, we adopt the McDonald result that RR Lyraes belonging to the two 0osterhoff groups have roughly the same $\left\langle M_{v}\right\rangle(=+0.55)$, and that MR-RR's are fainter than this by about $0.25 \mathrm{mag}\left(\left\langle M_{v}\right\rangle=+0.80\right)$.

The McDonald values of intrinsic $(B-V)$ provide also an independent technique for measuring the color excess $E(B-V)$ of clusters containing fairly metal-rich RR Lyraes, e.g., NGC 6712 and NGC 6171. For NGC 6712, we have $[\mathrm{Fe} / \mathrm{H}]=-0.4$, so the values of $\langle(B-V)\rangle_{\text {mean }}$ light and $\left\langle(B-V)_{\text {min. }}\right.$ light in Table 1 corresponding to MR-RR's apply directly. From the photometry of RR Lyraes by Sandage, Smith and Norton (1966), we have $\langle(B-V)\rangle_{\text {mean light }}=0.79 \pm 0.07$ (m.e.) and $\left\langle(B-V)_{\text {min. light }}=0.88 \pm 0.11\right.$ (m.e.), leading to $E(B-V)=0.42$ and $E(B-V)=0.43$, respectively, whereas from UBV photometry of field stars in the region of the cluster, Sandage and Smith (1966) obtained $0.44 \leqslant E(B-V) \leq 0.50$. We adopt $E(B-V)=0.44$, from which it follows that the intrinsic color at the junction 
of the giant (GB) and horizontal branches $(H B)$ is $(B-V)_{0, g}=0.94 \pm 0.06$ (est. m.e.). Similar precepts applied to the RR Lyrae photometry in NGC 6171 (Sturch 1967, Dickens 1970) lead to $\langle E(B-V)\rangle_{\text {min.light }}=\langle E(B-V)\rangle_{\text {mean light }}=$ $0.36 \pm 0.02$ (m.e.), on the assumption that RR Lyraes of NGC 6171 have intrinsic colors midway between those of MR-RR's and 0osterhoff group I. From UBV photometry of field stars, Dickens derived the somewhat smaller value of $E(B-V)=0.28$, and we adopt $E(B-V)=0.34$, from which it follows that $(B-V)_{0, g}=0.88 \pm 0.04$ (est. m.e.). We summarize the photometric and spectroscopic parameters for the four metal-rich clusters in Table 2; values of other metallicity parameters (Kinman 1959, Hartwick 1968, van den Bergh 1969) are also given. Plots of $(B-V)_{0, g}$ as a function of Kinman (1959) spectral type and $[\mathrm{Fe} / \mathrm{H}]$ are given in Figure 1 ; the latter is the same as Butler's (1975 diagram except for the substitution of our values of $(B-V)_{0, g}$ in the cases of the four clusters of Table 2. We conclude that, on the basis of spectral type, direct $[\mathrm{Fe} / \mathrm{H}]$-measurement, and the derived value of $(B-V)_{0, g}$, NGC 6712 has essentialiy the same metal abundance as $M 71$ and 47 TuC. Why then does NGC 6712, in contrast to $M 71$ and 47 TuC, have a number of RR Lyraes and a well-developed blue horizontal branch?

That this is not simply a "numbers effect" resulting from the selection of a very much smaller sample of stars in the cases of $M 71$ and 47 Tuc, as compared with NGC 6712, can be seen from inspection of Table 3. Photometry for the clusters is complete within a well-defined annulus centered on each cluster; the number of giants sampled in NGC 6712 exceeds that in the other two clusters by a factor of only 2. The relatively larger value of $n(H B) / n(R G)$ for NGC 6712 compared with $M 71$ and 47 Tuc cannot be explained as a statistical accident. 
Table 2.

Adopted Color Excesses $E(B-V)$ and $(B-V)_{0, g}$ for Metal-Rich Clusters

Cluster

\begin{tabular}{|c|c|c|c|c|c|c|c|}
\hline$N G C$ & $M$ & {$[\mathrm{Fe} / \mathrm{H}]$} & $E(B-V)$ & $\begin{array}{l}\text { Sp T } \\
\text { (Xinman } \\
1959)\end{array}$ & $(B-V)_{0, g}$ & $\begin{array}{c}S \\
\text { (Hartwick } \\
1968)\end{array}$ & $\begin{array}{c}L \\
\text { (van den Bergh } \\
1969)\end{array}$ \\
\hline 104 & 47 Tuc & -0.48 & $0.04^{3}$ & G3 & 0.96 & 3.4 & - \\
\hline 6838 & 71 & -0.35 & $0.29^{7}$ & G5 & 0.92 & 3.4 & - \\
\hline 6712 & - & -0.39 & $0.44^{2}$ & 64 & 0.94 & 4.5 & 8 \\
\hline 6171 & 107 & -0.82 & $0.33^{2}$ & GO-1 & 0.88 & 3.5 & 7 \\
\hline
\end{tabular}

Table 3.

Relative Star Counts in the HR Diagrams of NGC 6712, M 71, and 47 TUC

Cluster $[\mathrm{Fe} / \mathrm{H}] \quad \begin{gathered}\text { No. } \\ \text { Giants }\end{gathered} \underset{\text { Clump Stars }}{\mathrm{No}, \mathrm{HB}^{2}} \stackrel{\text { No. } \mathrm{HB}^{3}}{\text { Blue Stars }} \underset{\mathrm{RR}}{\mathrm{No}^{4}} \quad$ Photometry

$\begin{array}{ccccccc}\text { NGC 6712 } & -0.39 & 66 & 64 & 36 & 9 & \begin{array}{l}\text { Sandage \& Smith } 1966 \\ \text { Sandage, et al } 1966\end{array} \\ \text { M 71 } & -0.35 & 31 & 35 & 4 & 0 & \text { Arp \& Hartwick 1971 } \\ 47 \text { Tuc } & -0.48 & 31 & 17 & 0 & 0 & \text { Hartwick \& Hesser } 1974\end{array}$

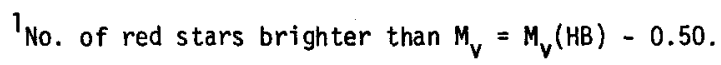

${ }^{2}$ No. of HB stars with $0.50 \leq(B-V) \leq 0.80$ (unreddened).

$3^{3}$ o. of $H B$ stars with unreddened $(B-V)<0.50$.

${ }^{4}$ Confined to the annulus in which photometry was carried out. NGC 6712 contains 10 RR Lyraes al together. 


\section{Mass, Temperature, and Luminosity of MR-RR's as a Constraint on}

\section{the He-abundance of the Old Galactic Disk}

\section{A) General Remarks on the He Abundance in the Galactic Disk}

In a highly provocative recent paper, Demarque and McClure (1977a) showed that two clusters having the same metal abundance (viz., $[\mathrm{Fe} / \mathrm{H}]=-0.5$ ) could nevertheless have HR diagrams of remarkably different morphology. One, the globular cluster 47 Tuc, we discussed above in a different context; the other is the old galactic (disk) cluster NGC 2420. From comparison with theoretical evolutionary isochrones, they concluded that the difference in morphology could not be explained in terms of a difference in age alone, but rather required an additional difference in chemical abundance amongst elements not contributing directly to the UV-excess (from which the $[\mathrm{Fe} / \mathrm{H}]$ value had been estimated). One of two conditions must evidently be satisfied: either (1) the helfum abundance of 47 Tuc is greater than that of NGC 2420 by $\sim 0.1$ in $Y$, or (2) the CNO abundance in NGC 2420 is lower than that in 47 Tuc by about an order of magnitude. Against the latter alternative is the observed fact that the $\mathrm{CN}$ band-strengths are similar in the spectra of stars of the two clusters (McClure and Osborn 1974; Hesser, Hartwick and McClure 1976; McClure, Forrester and Gibson 1974). Possible scatter in CNO-abundances complicates the issue, and we discuss this point more extensively later. The first solution, involving helium, seems preferred by the authors, but one notes that the consequence of its acceptance is somewhat startling: There is a disk galactic cluster with lower helium abundance than a globular cluster. As emphasized by the authors, the existence of NGC 2420 forces one to reconsider the view that heavy-element enrichment is a function of age alone, but rather depends 
also on position in the galaxy; indeed, the enrichment process may be quite spotty. A similar argument has been advanced by Sandage and Hartwick (1977) in connection with the metal abundance of the remote globular cluster Pal 5.

The age of NGC $2420\left(4 \times 10^{9} \mathrm{yr}\right)$ with the lower He abundance turns out to be less than half that of 47 Tuc. Low He-abundances also have been found in the stars of some still younger open clusters in the direction of the galactic anticenter (Nisson 1976). But of interest in connection with the problem of the MR-RR's is the possibility that some part of the older disk, viz., that stellar population containing the MR-RR's, has a helium abundance lower than that of the halo and nuclear globular clusters. A view of this kind was advanced some time ago by Gross (1973), who obtained He abundances for various galactic stellar populations by fitting theoretical to "observed" values of $T_{e}, L$, and $\log g$ for HB(SET) models. Gross concluded that globular cluster HB populations were consistent with $Y \geq 0.30$, but that old disk clusters had red HB "clump" populations requiring $Y \sim 0.23$; for the latter, errors in $Y$ proved large $( \pm 0.1$ to 0.2$)$ because of the inadequacy of relevant model atmosphere calculations, and Gross's conclusion remained therefore somewhat problematical.

However, armed with McDonald's new values of $\left\langle M_{v}\right\rangle$ and $\left\langle T_{e}\right\rangle$ for $M P$ and $M R$ RR Lyraes, and the evidence marshalled by Demarque and McClure in the case of NGC 2420, we suggest that MR-RR's of the disk have lower helium than RR Lyraes of globular clusters by about $\Delta Y \sim 0.07$; we show that this difference in $Y$ completely explains the differences in $\left\langle P_{a b}\right\rangle$ and luminosity. We combine the familiar results of pulsation theory and SET for the ZAHB (zero-age horizontal branch). All analytical expressions used are approximate; their authors warn 
the unwary regarding the limits of their applicability (cf. e.g., Iben 1974). I nevertheless adopt them here and use them, not absolutely, but relatively, to compare stars in one population with another.

According to Iben $(1971,1974)$, the pulsation equation is nearly independent of composition parameters. The fundamental period $P_{F}$, expressed in days is approximated by

$\log P_{F}=-0.340+0.825(\log L-1.7)-3.34\left(\log T_{e}-3.85\right)-0.63(\log 7+0.19)$

where luminosity $L$ and mass th are in solar units. (A similar formula given by van Albada and Baker [1973] has coefficients differing from those of Iben by $\leq 5 \%$. The small differences are irrelevant to the subsequent discussion, and we adopt the Iben formulation for illustrative purposes.) From the same source we also find

$\log L_{R R} \simeq 1.75+2.16(Y-0.3)-0.05(\log Z+3)+3.1(10, c-0.5)$

and

$\log \mathbb{N}_{\mathrm{RR}} \simeq-0.165+0.34(y-0.3)-0.076(\log z+3)+1.16(3) c^{-0.5)}$,

where $Y$ and $Z$ are the helium and metal abundances by mass, and $\mathbb{P}{ }_{c}$ is the mass of the helium care at the moment of the helium core flash. ${ }^{\mathbb{R}} \mathrm{c}$ depends on $y$ and $Z$, and we adopt the explicit dependences given in Figures 2 and 3 of Sweigart and Gross (1977). Strictly speaking, equations (2) and (3) apply only on the ZAHB, and it is likely that, at least in the more extreme ranges of metal abundance (i.e., $Z$ far from $Z(M)$ )), RR Lyraes are not strictly in the ZAHB-state. Although this is a weak point of the present discussion, it is hoped that evolutionary influences are diminished in an approach based on ratios of $L, T_{e}$, and $2 R$ in different populations. 
Consider any two populations of stars $A$ and $B$. We have from (1): $\log \frac{P_{F}(A)}{P_{F}(B)}=0.825 \log \frac{L(A)}{L(B)}+3.34 \log \frac{T^{(B)}}{T_{e}(A)}+0.63 \log \frac{\prod^{n}(B)}{(A)}$.

The third term on the right-hand side of equation (4) is a function essentially of $Y$ and $Z$ only. The second term, in principal, is a function of $Y$ and $Z$, but is also directly given by observation, if we adopt McDonald's (1976) results. The first term is given by observation and also is a function of $Y$ and $Z$, and the left-hand side is given directly by observation. The question is whether, given $Z(A)$, and $Z(B)$, one can find a value of $Y(B)-Y(A)$ consistent with the strong observational constraint on $\frac{P_{F}(A)}{P_{F}(B)}$, and the somewhat weaker observational constraints on $\frac{L(A)}{L(B)}$ and $\frac{T_{e}(B)}{T_{e}(A)}$.

B) Application to M 15 and M 3 (0osterhoff groups II and I, respectively)

To show that the equations make sense, we illustrate the application of (2) to (4) to the case $A=M 15$ (0osterhoff Type II) and $B=M 3$ (0osterhoff Type I). We assume $Y(M 15)=Y(M 3)$ and $\log Z(M 3)-\log Z(M 15)=(-3.1)-$ $(-3.7)=[\mathrm{Fe} / \mathrm{H}]_{M 3}-[\mathrm{Fe} / \mathrm{H}]_{M 15}=(-1.4)-(-2.0)=+0.6$. [The metal abundance of $M 3$ remains regrettably somewhat uncertain because of contradictory results derived from various equally valid methods. We adopt $[\mathrm{Fe} / \mathrm{H}]=-1.4$, a mean of various values quoted in Table 7 of Butler (1975a)]. For each of the three terms on the right-hand side of equation (4), we then have the following:

First term: $\quad \log \frac{L(M \quad 15)}{L\left(M \frac{3}{3}\right)}=+0.045 \rightarrow \Delta M_{v}=0.11$ mag.

Second term: $\log \frac{T_{e}(M 3)}{T_{e}(M T 5)}=+0.0143$, from $T_{e}$ 's in Table 1 .

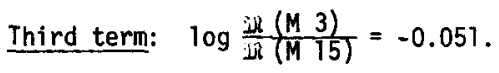


Combining the terms in equation (4), we obtain +0.053 for the right-hand side, to be compared with the observed value (left-hand side) of +0.066 . We would have obtained perfect agreement if the difference in $\langle T\rangle$ for the Bailey $a b$ 's in the two clusters were increased by only $60^{\circ}$, from $220^{\circ} \mathrm{K}$ to $280^{\circ} \mathrm{K}$. On the other hand, if we left the temperature term alone and simply changed the helium slightly, say $Y(M 3)-Y(M 15)=0.01$, we wowld obtain nearly perfect agreement between the two sides of equation (4). Recalling that McDonald's (1976) observations require that $M_{V}\left(R R_{M}\right) \approx M_{V}\left(R R_{M}{ }_{15}\right)$ (Table 1), we find that the choice $Y(M 15)=Y(M 3)$ is the more reasonable, since it leads to the smaller $\Delta M_{v}(0.11 \mathrm{mag} v s .0 .17 \mathrm{mag})$. In any case, the agreement between the theoretical prediction and the observations is entirely satisfactory and gives confidence that the extrapolations of subsections (C) and (D) are reasonable.

C) MR-RR's of the Solar Vicinity vs. NGC 6712

We now apply equation (4) to the case in which $A=$ MR-RR's of the solar vicinity, denoted by "(disk)" hereinafter, and $B=$ metal-rich RR Lyraes of NGC 6712. We take $Z_{\text {(disk) }}=Z_{(6712)}$, and express terms (1) and (3) of equation (4) as functions of $\left[Y_{(d i s k)}-Y_{(6712)}\right]$. Interpolation in Figure 2 of Sweigart and Gross (1977) for $Z$ fixed yieids $I_{c}=0.525-0.2 Y$, so that equation (4) then becomes:

$\log \frac{P_{F}(\text { disk })}{P_{F}(6712)}=0.825\left[1.53\left(Y_{(\text {disk })}-Y_{(6712)}\right]+3.34 \log \frac{T_{e}(6712)}{T_{e}(\text { disk })}+\right.$

$$
0.63\left[-0.11\left(Y_{(\text {disk })}-Y_{(6712)}\right)\right] \text {. }
$$

With $\log \frac{P_{F} \text { (disk) }}{P_{F}(6712)}=\log \frac{0.45}{0.56}=-0.095$ given by observation, what should we 
adopt for $\log \frac{T_{e^{(6712)}}}{T_{e}{ }^{(d i s k)}}$ ? Although $T_{e}$ (disk) is known from McDonald's (1976) work, there is no way to determine $T_{e}(6712)$ directly. We know that $T_{e}(6712)$ cannot differ much from $T_{e}$ (disk), otherwise the correlation between $(B-V)_{0, g}$ and $[\mathrm{Fe} / \mathrm{H}]$ would be disturbed (cf. Fig. 1 and Table 2). We consider two cases: Case I. $T_{e}(6712)=T_{e}$ (disk). The second term of (5) then drops out and we obtain $Y_{\text {(disk) }}-Y_{(6712)}=-0.080$.

Case II. According to Iben (1971, Figure 18), the temperatures $T_{F B E}$ and $T_{H B E}$ of the fundamental and first harmonic blue edges, respectively, are independent of $Z$ and depend only on $Y$ and $L$. We assume $\frac{T_{e}(6712)}{T_{e}(\text { disk })} \approx \frac{T_{F B E}(6712)}{T_{F B E}(\text { disk })} \approx \frac{T_{H B E}(6712)}{T_{H B E}(\text { disk })}$, expressing the dependence of the ratio of the temperatures on $L$ explicitly through Fig. 18 of Iben (1971), and then the dependence of $L$ on $Y$ as in the first term of equation (5) above. Using $T_{F B E}$, we find $\log \frac{T_{e^{(6712)}}}{T_{e^{(d i s k)}}}=0.089\left(Y_{(\text {disk })}-Y_{(6712)}\right)$, whereas using $T_{H B E}$, we obtain $\log \frac{T_{e} e^{(6712)}}{T_{e}(\text { disk })}=0.191\left(Y_{(d i s k)}-Y_{(6712)}\right)$. Solving for $\left(Y_{(\text {disk })}-Y_{(6712)}\right)$ using equation (5) we find $\left(Y_{(\text {disk })}-Y_{(6712)}\right)=-0.064$ and -0.052 , with $T_{e}$ (6712) lower than that of $T_{e}$ (disk) by $95^{\circ} \mathrm{K}$ and $155^{\circ} \mathrm{K}$, respectively. To remove entirely the change in $Y$ between (disk) and NGC 6712 would require $\mathrm{T}_{\mathrm{e}}$ (disk) $-\mathrm{T}_{\mathrm{e}}(6712) \approx 400^{\circ} \mathrm{K}$, which, according to model atmosphere calculations (Phillip 1977), would correspond to $\Delta(B-V) \approx 0.10$ for horizontal branch stars with $Z \sim 10^{-2}$ and $T_{e} \sim 6700^{\circ} \mathrm{K}$. This result would completely upset the location of NGC 6712 in the plot of $(B-V)_{0, g} \underline{\text { vs. }}[\mathrm{Fe} / \mathrm{H}]$ (Figure 1), and seems inadmissable. 


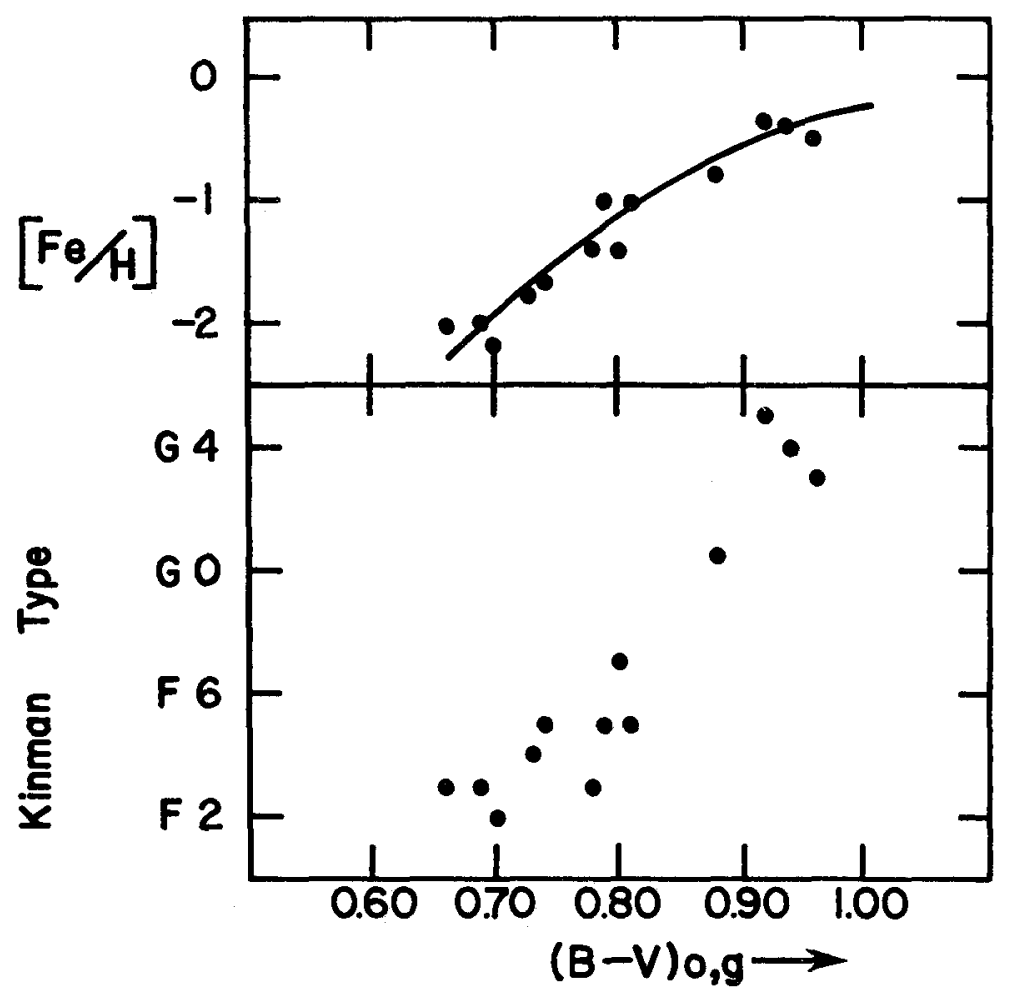

Figure 1. $[\mathrm{Fe} / \mathrm{H}]$ and Kinman-type as a function of $(B-V)_{0, g}$ for 14 globular clusters (after Butler 1975 
The analysis above, subject to the uncertainty in the ratio $T_{e}$ (disk)/ $T_{e}(6712)$, strongly suggests that we can attribute the difference in mean period of the Bailey ab's to a difference in helium abundance of about $Y_{(\text {disk })}-Y_{(6712)} \approx-0.07$, with an outside uncertainty of about \pm 0.03 . This requires that the temperature of MR-RR's in the solar vicinity should be about $100^{\circ} \mathrm{K}$ lower than those in NGC 6712 (corresponding to an acceptable $\Delta(B-V) \sim 0.02$ ), and that they should also be fainter by $\Delta M_{v}=0.27$ mag.

\section{D) MR-RR's (disk) vs. M 3}

We close the circle finally by comparing the metal-rich RR Lyraes in the solar vicinity with those in M 3. Here, the first two terms of equation (4) are derivable empirically (Table 1), but the first term can also be written as a function of $Y$ and $Z$. From Table $I$, we have:

First term: $\quad 0.825 \log \frac{L(M 3)}{L(\text { disk })}=+0.082$

$$
\text { Second term: } \quad 3.34 \log \frac{T_{e} \text { (disk) }}{T_{e}(M 3)}=+0.008
$$

The third term can be evaluated from inspection of the HB-SET tracks given by Sweigart and Gross (1976), from which we find $\mathbb{R}_{R R}(M 3) \sim 0.65^{\mathfrak{R}}$, whereas $\mathbb{I l}_{R R}$ (disk) $\sim 0.60 \mathbb{D}_{\theta}$. Thus the right-hand side of equation (4) becomes +0.068 , whereas the observed value of the left-hand side is +0.087 . The agreement thus is quite good, and would be exactly right if we had taken a temperature difference of $100^{\circ} \mathrm{K}$ rather than $35^{\circ} \mathrm{K}$.

With $\log \frac{L(M 3)}{L(\text { disk })}=+0.11$ from observation, we can also write $\log \frac{L(M 3)}{L(\text { disk })}=2.16\left(Y_{(M ~ 3)}-Y_{(\text {disk })}\right)-0.05[\log Z(M 3)-\log Z$ (disk) $]+$ $3.1\left(\mathcal{L}_{c}\left(M_{3}\right)-M_{c(\text { disk })}\right)$. Interpolating the latter term in Sweigart and 
Gross (1977, Fig. 3) as a function of $Y$ and $Z$; we have ${ }^{\nu l} c(M 3)-\mathbb{R}_{c}$ (disk) $=$ 0.0 when $Y_{(M ~ 3)}-Y_{(\text {disk })} \approx+0.07$; whence $\log \frac{L(M 3)}{L(d i s k)}=+0.15$, which is very close to the value required by the observations.

E) Self-consistent Abundance, Luminosity, and Temperature Differences between RR Lyraes of the (disk), NGC 6712, and M 3

We now summarize the doubtless rather confusing detatls of paragraphs (A) through (D) of this section. We begin by adopting abundances, luminosities $L$, and effective temperatures $T_{e}$ for RR Lyraes in $M 3$, and estimate the corresponding quantities for NGC 6712 and (disk) from the discussion above. According to Iben, there is a relationship between $Y$ and $T_{e}(H B E)$ which for $M 3$, where $\log P_{\text {HBE }}=-0.55$, is

$Y=0.22+5\left(\log T_{e}(H B E)-3.863\right)$.

From Sandage (1969) we have $(B-V)_{H B E}=0.18$ for $M 3$, so that in accordance transformations given by Philip (1977), we find $(b-y)_{H B E}=0.14$ and $\log T_{e}(H B E)=3.872$. Then $Y=0.265$ from equation (6). For the same stars, McDonald gives $\log T_{e}(\mathrm{HBE})=3.868$, and thus $Y=0.245$. Other methods for obtaining $Y$ have been discussed extensively in the literature (cf. e.g., Iben 1974, Renzini 1977), and range from 0.18 to 0.38 . All are sensitive to input parameters and to the physics of the assumptions. We have mentioned already Gross's (1973) finding that $Y($ halo $)>0.30$. We adopt here the value $Y(M 3)=0.27$, based largely on our approach via RR Lyraes, but emphasize the importance in our discussion of the relative value of $Y$ between various families of RR Lyraes, not the absolute value. 
Table 4 gives the best relative values of $X, Y, Z,\left\langle M_{v}\right\rangle$, and $\langle T\rangle$ for NGC 6712 and (disk) based on values assumed for M 3, and the discussion in subsections $(A)$ to $(D)$.

Table 4.

"Best-Fit" Parameters

\begin{tabular}{|c|c|c|c|c|c|}
\hline $\begin{array}{l}\text { Cluster } \\
\text { or Group }\end{array}$ & $x$ & $Y$ & $\log z$ & $\begin{array}{c}\left\langle M_{v}\right\rangle_{\text {mean light }} \\
(\text { Bailey ab) }\end{array}$ & $\begin{array}{r}{ }^{<T_{e^{\prime}}} \text { mean light } \\
\text { (Bailey ab) }\end{array}$ \\
\hline M 3 & 0.72 & 0.27 & -3.2 & +0.55 & 6790 \\
\hline NGC 6712 & 0.71 & 0.27 & -2.2 & +0.60 & 6925 \\
\hline $\begin{array}{l}\text { MR-RR Solar } \\
\text { Vicinity }\end{array}$ & 0.78 & 0.20 & -2.2 & +0.85 & 6825 \\
\hline
\end{tabular}

The conclusion we reach is essentially this: if there exists a fairly metal-rich $(\log Z \sim-2.2)$ component of the old galactic disk having helium abundance Y lower than that of globular clusters by $\sim 0.07$, it will contain RR Lyraes having Tuminosities $\sim 0.3 \mathrm{mag}$. fainter and periods $20 \%$ shorter than those of RR Lyraes belonging to Oosterhoff's Type I, as required by observation. We now examine the ages and expected mass-loss of such stars, and inquire about their immediate giant-star metamorphs. The latter, which then must have low helium as well, are presumably all or part of the ODG population. Using the mass-loss theory of Fusi-Pecci and Renzini (1975), we first examine the consequences of a changed $Y$ on the total mass-loss. If an ODG were able to metamorphoze into a MR-RR with an alacrity like that of its globular cluster counterpart, the sky would be full of bright MR-RR's! Since this is clearly 
not the case, age and total mass-loss uil must conspire to limit the population. If we then calculate $\Delta$ ill, we can see what restriction this sets on the age of old disk stars with low $Y$.

\section{Mass-Loss for ODG's as a Function of $Y, Z$ and Age}

We consider the mass lost by a red giant after leaving the main sequence at a given turnoff luminosity $\left(L_{T} .0.\right)$ and mass $\left({ }_{R G}\right)$. As a function of $X$ and Z, Fusi-Pecci and Renzini (FP-R) (1975) show that the helium core flash luminosity $\left(L_{F l}\right)$ and flash mass $\left({ }^{2} F_{F l}\right)$ are given by the simultaneous solution of the equations:

$$
\begin{aligned}
& \mathbb{m}_{F i}^{2.67} \mathbb{R}_{R G}^{2.67}-0.0877 n\left(\frac{Z}{10^{-3}}\right)^{-0.08}\left(\frac{X}{0.7}\right)^{1.36} L_{F i}^{1.08} \\
& \log L_{F 1}=3.31+0.09(\log Z+3)+0.045(X-0.7)-0.157\left({ }^{18}{ }_{F 1}-0.8\right),
\end{aligned}
$$

where $n$ is an efficiency factor giving the fraction of convective energy needed to accelerate coronal matter to infinity. Observations of the solar wind flux suggest $n=7.5 \times 10^{-4}$ and we adopt this value in what follows. We solved the FP-R equations with $\mathbb{R}_{R G}=0.70,0.80,0.90,1.00 \mathbb{R}_{\theta}$ for the following choices of $Y$ and $Z$ :

$$
\begin{aligned}
& \text { 1) } Y=0.27, \log Z=-3.2 \text { (high helium, } Z=Z(M 3)=Z \text { (0ost. I)) } \\
& \text { 2) } Y=0.27, \log Z=-2.2 \text { (high helfum, } Z=Z(6712)) \\
& \text { 3) } Y=0.20, \log Z=-2.2 \text { (low helfum, } Z=Z \text { (MR-RR)) } \\
& \text { 4) } Y=0.20, \log Z=-2.6 \text { (low helium, } Z<Z_{\mathrm{Fe}}(\text { MR-RR)). }
\end{aligned}
$$

The coeffcients of equations (7) and (8) are explicitly tied to the red giant model calculations of Rood (1972); we have not attempted to recalculate the coefficients using the most recent models (Sweigart and Gross 1977). From 
the same source (Rood 1972), Renzini (1977) quotes the following useful analytical expressions for age $\left(t_{g}\right)$ (in billions of years) and ${ }_{R G}$ as functions of $X, Y, Z$ and $L_{T .0 .}$ :

$$
\log t_{9}=1.42-1.1 \log L_{T .0 .}-0.59(Y-0.3)-0.14(3+\log Z)
$$

and

$\log \mathbb{P}_{R G}=-0.29 \log t_{g}+0.75 x-0.0094(4+\log z)+0.0194(4+\log z)^{2}-$

For each value of $\mathrm{IR}_{R G}$ and choice of input abundance parameters (cases (1) (4)), we calculate $\log t_{9}$. On the other hand, any real globular or galactic cluster has an "observed" value of $\log L_{T .0}$, thus an age and ${ }^{2 n} R G$ via equations (9) and (10). We then ask: Is the flash mass $\mathbb{B}_{F}$ obtained from the "observed" $L_{T .0}$. and the solution of equation (7), which must then be the mass appropriate to the ZAHB of the cluster, compatible with the mass-range required by HB-SET [cf. e.g.. Sweigart and Gross (1976)] in the part of the HB occupied by RR Lyraes? If so, the cluster will presumably pump the RR Lyrae domain. 1

In Figure 2, we show the results in a form similar to that already given by FP-R (Figure 5) who calculated the two cases $Y=0.30$ with $\log Z=-1.7$ and -4.0 , Obviously, the older the age, the greater is the mass loss $\Delta \mathbb{R}=\mathbb{R}_{R G}-\mathbb{M}_{F l}$; for a fixed $Y$ and a given value of $\mathbb{L}_{R G}$, the mass-loss is

\footnotetext{
We ignore in all this the small difference between the mass of the star at cluster turn-off and the mass of the star actually arriving at the RG-tip at the same instant (in the absence of all mass-loss considerations). This difference does not exceed $\sim 0.01 \mathfrak{R}_{\theta}$ in any case (cf. e.g., Renzini 1977).
} 


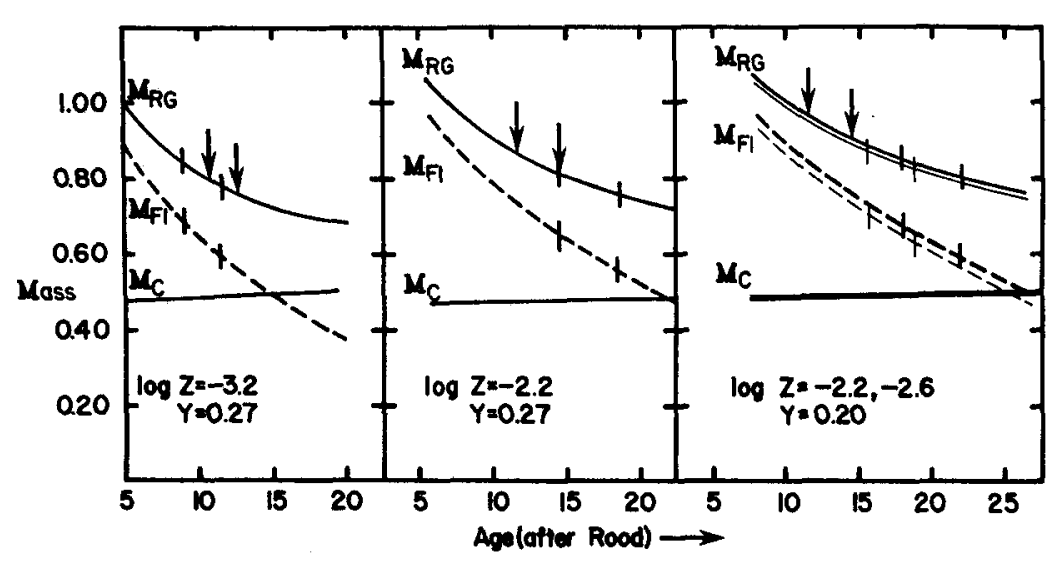

Figure 2. Turn-off Mass $\left(\mathfrak{i l}_{R G}\right)$, Flash Mass $\left(\mathcal{H}_{F l}\right)$ and Core Mass ( $\mathbb{I I}_{c}$ ) at the flash as functions of Age ( $t_{g}$, after Rood), for various choices of $Y$ and $Z$. In all cases, the efficiency factor $n=7.5 \times 10^{-4}$. In the left-hand panel, the left- and right-hand arrows give the age for M $3(\gamma=0.27)$, according to the calculations of Rood and of Demarque and Mcclure, respectively. In the other two panels, left- and right-hand arrows correspond to the oldest possible age (i.e., $t_{9}$ for $M 15$, with $Y=0.27$ ), according to the calculations of Rood and of Demarque and McClure, respectively. In the extreme right-hand panel, thick and thin lines refer respectively to $Z=-2.2$ and -2.6 .

Tick-marks give the interval of mass in which RR Lyraes will be found for the appropriate $Y$ and $Z$, according to SET. 
slightly greater as the metal abundance is increased, but this becomes noticeable $\left(>0.01{ }^{2}{ }_{0}\right)$ really only when $\mathbb{I R}_{R G} \geq 0.8 \mathrm{Dl}_{\theta^{*}}$. However, at fixed $Y$ and $t_{g}, \Delta^{\mathbb{P}}$ is larger when $Z$ is smaller; this is because, as $Z$ decreases, the input mass $\mathbb{I}_{R G}$ at a fixed age is smaller (see discussion by Renzini 1977). Similarly, if we fix $Z$ and lower $Y$, then we must go to a considerably older age to achieve the same mass-loss. This is because lowering $Y$ decreases the central temperature and requires therefore a larger mass at a fixed age or an older age at a fixed mass to achieve the same evolutionary state. This effect is partly offset by the slightly higher $L_{F 1}$ achieved for stars with lower $Y$ : the value of $\Delta \mathbb{R}$ is quite sensitive to the value of $L_{F l}$, since most of the mass is lost near the RG-tip.

As pointed out by FP-R, there is a limiting age $\tau_{g}$ for the production of a star on the ZAHB that is given when ${ }^{i R} \mathrm{Fl}$ reaches the core mass ${ }^{\mathscr{H}} \mathrm{c}$. As $Y$. decreases, $\tau_{9}$ increases; for a fixed $Y, \tau_{9}$ decreases as $Z$ decreases, largely because of the slight increase in $\mathbb{M}$. For cases (1) to (4) above, the values of $\tau_{g}$ are $15.0,21.5,26.5$, and 25.0, respectively. Since case (1) applies to M 3, it sets a fairly stringent upper limit on the age of the halo clusters; $\tau_{9}$ would be shorter if $Y$ were increased. The other cases do not provide useful age limits on their respective populations if all stars are assumed to be not older than those of $M 3$. 
IV. A Comparison of Sifl with the Mass-Range of RR Lyraes Expected from

\section{SET for Clusters with Given $Y, Z$ and Age}

In Table 5, we summarize, on the basis of the discussion of FP-R and equations (9) and (10) from Rood, the values of $t_{9}, \mathbb{I}_{R G}$ and $\nu_{F}$ expected for stars in globular and galactic clusters of various chemical compositions. We comment on the resuits as follows (cf. also Fig. 2):

1) M 15, M 3, Oosterhoff I and II Clusters. if $F 1$ is in the correct range for halo populations $(Z \leq-2.7)$ having high helium (Sweigart and Gross 1976). We find $\Delta, \mathbb{R}=0.2 \mathbb{H}_{0}$ and ages of 11 to $12 \times 10^{9} \mathrm{yr}$. Since $M 3$ has 2 slightly lower than the typical value $(z \sim-2.7)$ for an 0osterhoff Type I cluster, we calculate $\mathbb{R}_{F}$ also for $Z=-2.7$ and a fainter $L_{T .0}$, closer to that of $M 13$.

2) Metal-Rich Globular Clusters, e.g., 47 Tuc, M 71 . We assume $M_{v}(H B)=+0.5( \pm 0.1)$ corresponding roughly to MR-RR's of high helium; this assigns in a completely empirical way a value of $\log L_{T} .0$. independent of any consideration about the location of the ZAMS as a function of $Y$ (and $Z$ ). For $Y=0.27$, ages of 47 Tuc and $M 71$ are then 10.2 and $9.1 \times 10^{9} \mathrm{yr}$., respectively; an increase of $M_{v}(H B)$ by only 0.15 mag., from +0.5 to +0.65 , would increase the ages of these clusters by $\sim 15 \%$, thus making their ages equal to that of the halo clusters. Reckoned in units of $10^{10}$ years, there is no demonstrable difference in age between halo and nuclear globular clusters.

From the HB evolutionary tracks of Sweigart and Gross (1976) for $Z=0.01$ and $Y=0.30$, we estimate that, for the abundance parameters assumed here, stars must have masses in the range 0.56 to 0.66 $\mathbb{R}_{\text {g }}$ to occupy the instability strip. Thus 47 Tuc and $M 71$ giants lose insufficient mass to pump the RR Lyrae domain. Even with an age as old as that of $M 3$, these stars would still fail to metamorphose into $R R$ Lyraes by a margin of $\sim 0.07 \mathbb{R}_{\theta}$. 
3) Disk Galactic Clusters. [NGC 188 (high helium?), NGC 2420 (low helium)]. Regardless of the choice of $Y$, the projected values of ${ }^{\Perp R} \mathrm{Fl}$ are too large to permit occupation of the RR Lyrae domain.

4) NGC 6712 (high helium), MR-RR's of the Disk (low helium). We have no way of obtaining the ages of these populations directly since $L_{T .0}$. is unknown (photometry for NGC 6712, for example, does not extend below $M_{v} \sim+1.5$ ). If NGC 6712 has $t_{9} \approx 11.5$ (as old as $M 15$ ), then $L_{T .0 .}{ }^{(6712)}=L_{T .0 .}(47$ Tuc). If $t_{g}=11.5$ is the oldest possible age, then $\omega_{F l}=0.78 \|_{\theta}$, a value too large to produce RR Lyraes since the expected mass range is 0.56 to 0.66 il . Similar comments apply to MR-RR's, and the matter is made worse if $Y=0.20$ since ${ }^{4 l} \mathrm{Fl}$ then increases to $0.84 \mathfrak{R}$. However, with lowered $Y$, the limits of the expected mass-range increase somewhat because the helium core mass ${ }^{1 / l} \mathrm{C}$ increases with decreasing $Y$ (see Fig. 2) [the location of a star on the HB branch is sensitive to $\mathbb{M} / \mathbb{m}_{C}$, as shown long ago by Faulkner $\left.(1966)\right]$. Since Sweigart and Gross do not give evolutionary tracks for $Y=0.2$ and $Z=0.01$, we have had to estimate the size of the effect by comparing HB-SET tracks for different $Y$, with $Z=0.001$. We were guided to some extent also by the ZAHB calculations of Gross (1973, Figure 2) for $Z=0.01$. Thus, the mass-range quoted in Table 5 for the MR-RR case must be regarded as even more appropriate than the ranges given for the other entries.

In summary, we see that, for $Y=0.27$ in the case of NGC 6712, the mass discrepancy is at least $\sim 0.12 \mathscr{U R}_{\theta}$, and for disk MR-RR's with $Y=0.20$ it is at least $\rightarrow 0.16 \mathfrak{R}_{\theta}$, both for the oldest possible age $\left(t_{g} \sim 11.5\right)$. The fact that very few RR Lyraes are produced per ODG progenitor (Taam, et al. 1976) seems compatible with this result, but we are left with no obvious source for RR Lyraes in the case of NGC 6712. The best we can say is that if some giants of NGC 6712 succeed in pumping the RR Lyrae domain, the periods will be $20 \%$ Tonger and the luminosities $10 \%$ brighter than for MR-RR's of the solar vicinity. 


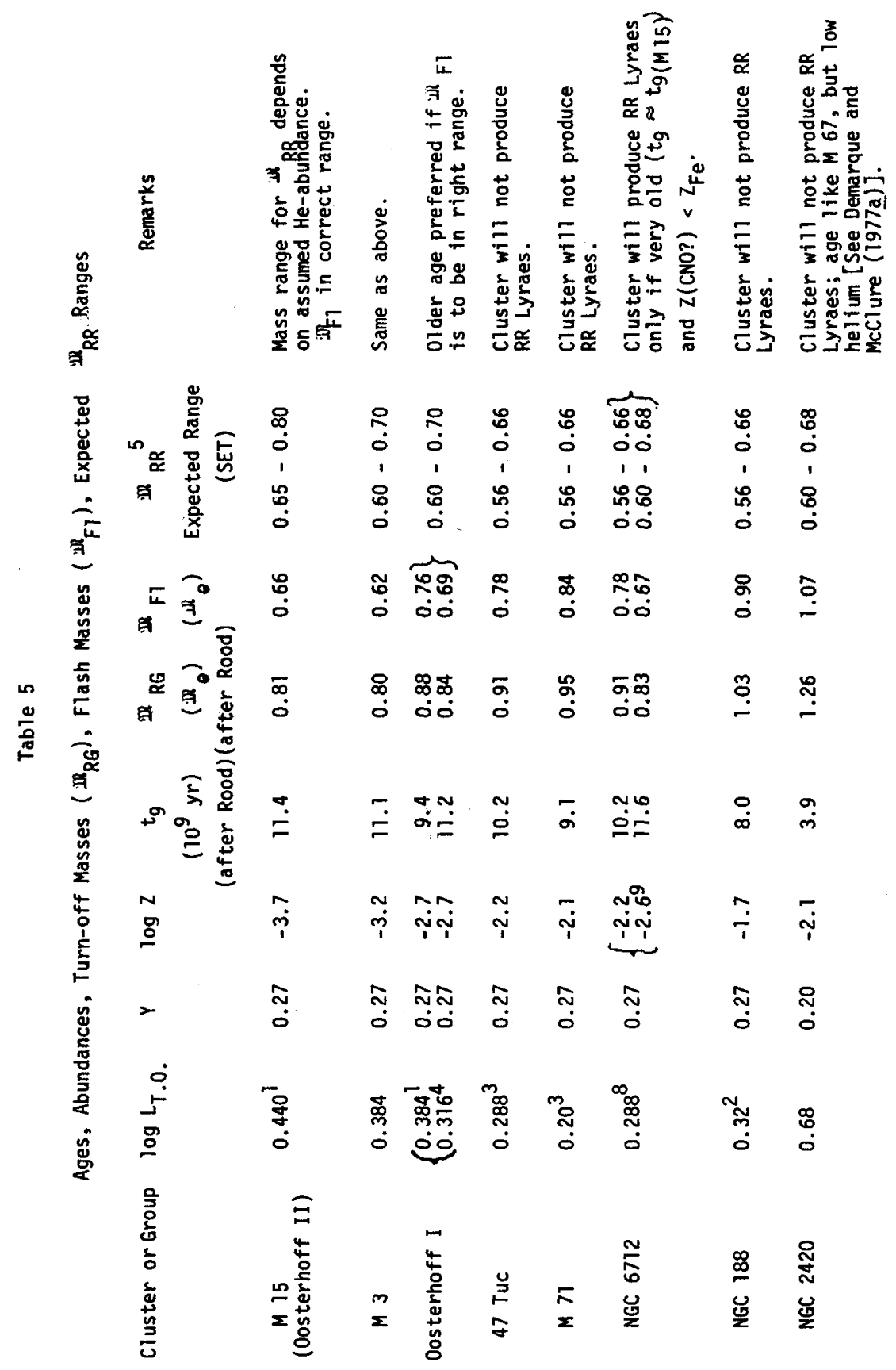




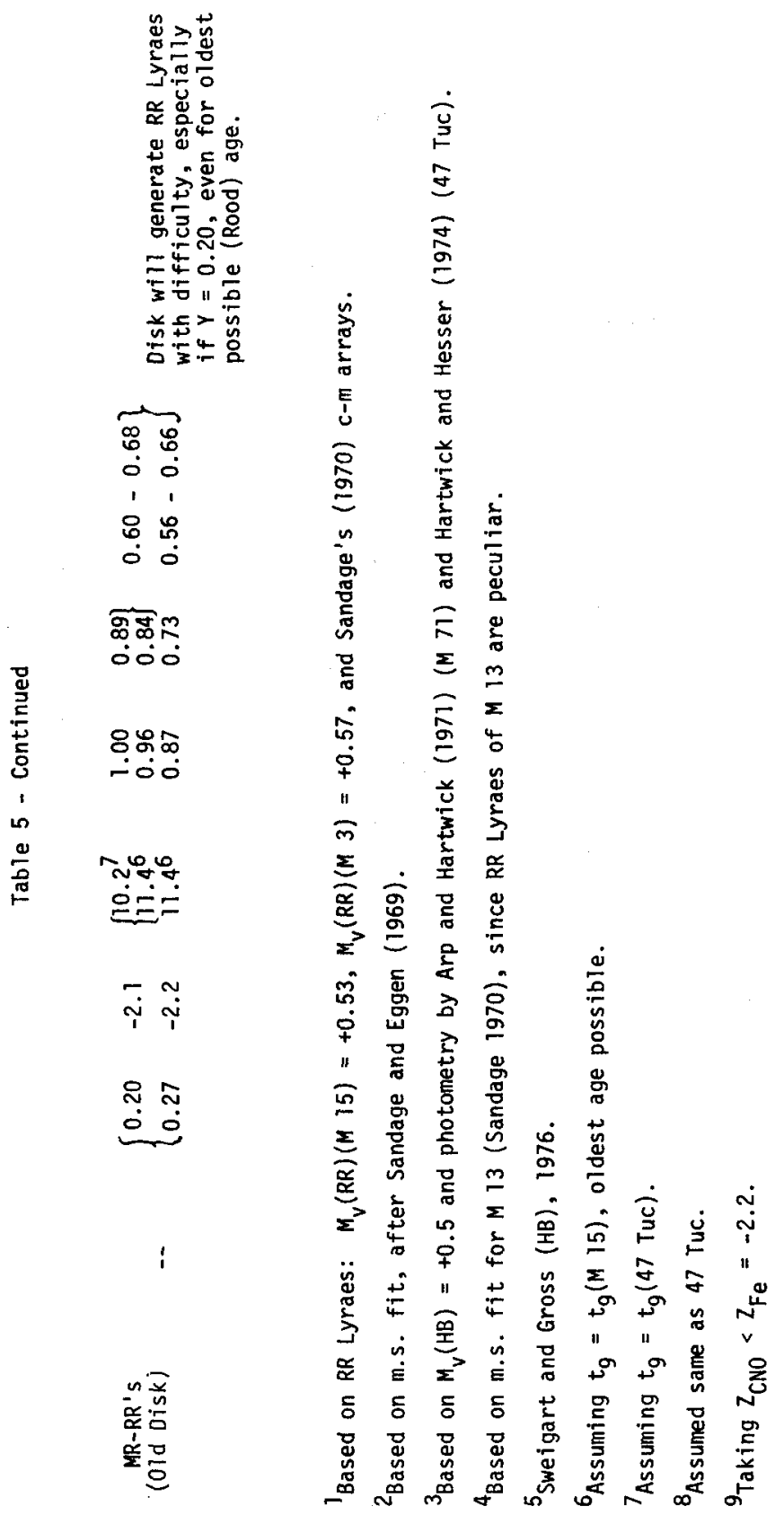




\section{Limitations, Problems, and Successes}

We have seen how the assumption that the galaxy contains an old disk component with low helium gives the desired observational properties, viz., short period and low luminosity, for metal-rich RR Lyraes of the solar vicinity. But the problem of the actual production of such stars remains, unsolved, at least within the framework of HB-SET (Swetgart and Gross 1976) and the scaled solar-wind mass-loss theory of Fusi-Pecci and Renzini (1975). The latter may, of course, be inapplicable or too rigidly applied here; nevertheless, it is of interest to explore how far one can go in easing the problem of the production of MR-RR's using the existing formulation. Each of the following proposals may therefore be regarded as a modest deus ex machina, by whose intervention MR'RR's are generated in NGC 6712 but not in the solar vicinity.

1) $t_{g}(\max )=t_{g}(6712)$ is too short. We adopted the ages given in earlier sections on the basis of Rood's models (after Rood 1972, Renzini 1977); more recent models lead to $t_{g}(\max )=t_{g}(M 15)=14.5$ for $Y=0.27$, about $25 \%$ longer (Demarque and Mcclure 1977b). If, for these older ages, $\mathbb{R}_{R G}$ scales as in equation (10), $\mathbb{R}_{F 1}$ reaches the domain of RR Lyraes in the case of NGC 6712 , provided the cluster is essentially as old as M 15. However, even if the disk were as old as these clusters, $D_{F 1}$ would still not reach the required mass interval if $Y=0.20$ (cf. Fig. 2). This solution thus has the obvious advantage that it produces RR Lyraes in NGC 6712 with ease, but not in the field. On the other hand, we must not make $t_{9}$ so large that the giants of $M 3$ and $M 15$ skip the production of RR Lyraes altogether. 
2) $\mathrm{Z}_{\mathrm{CNO}} \neq \mathrm{Z}_{\mathrm{Fe}}$. We have so far tacitly assumed that the metal abundance of the crucial CNO group is the same as that of the Fe-peak metals from which the observed values of $Z$ are actually estimated. For stars at the turn-off and on the $H B$, the crucial $Z$ is really $Z_{C N O}$ [cf. arguments by Iben (1974), Demarque and MCClure (1977 $\underline{b}$ ), Renzini (1977), and many others], since the CNO abundances control the opacity and the energy generation of the hydrogen shell source. There is now a growing body af evidence not only that $Z_{\mathrm{Fe}} \neq Z_{\mathrm{CNO}}$, but also that there is a range in CNO abundance, presumably primordial, in some globular clusters (Hesser, Hartwick, and McClure 1976; Carbon, Langer, Butler, Kraft, Nocar and Kemper 1978). Langer (1977) has also shown that the giant branch color-spread of M 92 can be explained entirely by the observed spread in CNO abundance (Carbon, et al. 1978). We could then postulate that for NGC 6712, $\mathrm{Z}_{\mathrm{CNO}}<\mathrm{Z}_{\mathrm{Fe}}$, or alternatively that some fraction of giants in NGC 6712 have a reduced value of $Z_{\text {CNO }}$. We show in Table 5 that, in NGC 6712 , a reduction of $Z$ from -2.2 to -2.6 is sufficient to produce RR Lyraes even if the age is the oldest possible on the (shorter) time-scales of Rood, of course $Z_{\text {CNO }}$ may not equal $Z_{\mathrm{Fe}}$ in the MR-RR's of the disk, either, but if the spread in $Z_{\text {CNO }}$ were the same in the two populations, one could easily discriminate against the disk by a slight decrease in disk age. Clearly the efficiency of the pump is very sensitive to small changes in age and/or $\mathrm{Z}_{\mathrm{CNO}}$.

3) Variations in $n$. The efficiency parameter $n$ is evaluated empirically from the solar wind mass-loss rate. Increases in $n$ by $20 \%$ do not affect $\Delta \mathbb{R}$

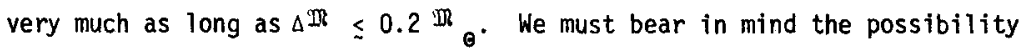
that $n$ could vary with $Y$, but the writer is incapable of deducing whether this is a possibility. 
VI. Other Evidence for Low Helium Within the Old Disk Population (ODP)

Some of this evidence has been reviewed by Gross (1973); we confine attention here to three representatives of the ODP, viz., ODG's, planetary nebulae, and old disk dwarfs (ODD's).

1) Colors of ODG's. For the globular cluster giants in NGC 6712, M 71, and 47 Tuc, we find from Table 2 that $\left\langle(B-V)_{0, g}\right\rangle=+0.94 \pm 0.02$ (m.e.) and $\langle[\mathrm{Fe} / \mathrm{H}]\rangle=-0.4 \pm 0.1$ (m.e.). These stars have $\left\langle M_{v}\right\rangle \equiv+0.5$, and presumably $Y=0.27$. The main body of $\mathrm{ODG}^{\prime} \mathrm{s}$, however, has $\langle[\mathrm{Fe} / \mathrm{H}]\rangle=-0.2$, f.e., is slightly more metal rich and we assume here that $Y=0.20$ for these stars. According to the red giant atmospheric models of Bell, Eriksson, Gustafsson, and Nordlund (1976), an increase of $[\mathrm{Fe} / \mathrm{H}]$ by 0.2 should redden $(B-V)$, owing to increased selective line blocking, by 0.03 . But the giant branch position also changes in addition because of interior effects: these are a function of $Y, Z$, and at a fixed $L$. We consider $M_{V}=+0.5$ corresponding to $\log L / L_{0}=1.80$ or $M_{b o 1}=+0.2$, and ignore the small change in B.C. with $T_{e}$.

For this $L / L_{\theta}$, we estimate the effect on $\log T_{e}$ if we decrease $Y$ by 0.07 and increase $\mathbb{R}$ from $0.91 \mathbb{R}_{\ominus}$ (the $\mathbb{M}_{R G}$ for $47 \mathrm{Tuc}$ ) to $1.00 \mathbb{R}_{\theta}$ (the $\mathbb{M}_{R G}$ for MR-RR's having $Y=0.20$ ) (Table 5). The most recent red giant models of Sweigart and Gross (1977) have a grid of $i, Y$, and $Z$ sufficiently fine to make such an estimate possible. For $\log L / L_{\theta}=1.80$, we find:

and

$$
\begin{aligned}
& Y=0.20, \text { Di }=1.00 \mathbb{H}_{\dot{\theta}}, Z=-1.9+\log \mathrm{T}_{e}=3.600, \\
& Y=0.27, \mathbb{M}_{i}=0.91 \mathbb{M}_{0}, Z=-2.1 \rightarrow \log \mathrm{T}_{e}=3.613 .
\end{aligned}
$$

Roughly, an increase of 0.2 in $\log Z$ is equivalent to a decrease in $Y$ of 0.1 . Thus for the change in question, about $1 / 3$ comes from $\Delta Y$ and $2 / 3$ from $\Delta Z$. We 
compute $\delta T_{e}=-140^{\circ} K^{\star}$, which corresponds to an increase in (B-V) of 0.09 . Adding this to the 0.03 reddening produced by line blocking yields a total increase in $(B-V)$ of 0.12 , so we predict that the ODG branch should have a color $(B-V)=+1.06$ at $M_{V}=+0.5$, if $(B-V)_{0, g}=+0.94$ for the metal-rich globular clusters. The actual observed value (Tinsley and Gunn 1976) is $(B-V)=+1.08$, and the agreement is very satisfactory.

It is important to note that the increase in ${ }_{k G}$ with decreasing $Y$ tends to reduce the effect: $i . e .$, as $D_{R G}$ increases, $T_{e}$ increases, everything else being equal. Roughtly $\left(\frac{\delta \log T_{e}}{\delta \text { it }}\right)_{L, Y, Z} \approx+0.05$. If the disk is made too young, ${ }_{R G}$ will increase, and the giant branch will move blueward. Thus the good agreement found here between the observed and predicted difference in color of the ODG's and the globular cluster giants (at a fixed $L$ ) supports the notion that the disk is old, and that the progenitors of ODG's do not exceed $\mathbb{P}_{\mathrm{RG}} \sim 1.00 \mathbb{M}_{\mathrm{\theta}}($ for $Y=0.20)$.

The $M_{v}$ vs. (B-V) diagram of Wilson (1976), in which $M_{v}$ is based on $K_{2}$ emission-line strengths, shows that, although the average star with $M_{V} \sim+0.5$ has $(B-V)$ of $\sim+1.10$, there exists a significant fraction of giants with $(B-V)>1.20$. Some of these no doubt have an interstellar reddening of a few hundredths of a magnitude (Eggen 1973a). The balance may be explicable on the basis of increased line blanketing due to abundances $[\mathrm{Fe} / \mathrm{H}]$ ranging up to $\sim+0.1$.

\footnotetext{
ॠThe values of $\mathrm{T}_{\mathrm{e}}$ given by $\mathrm{SW}(1977)$ are about $600^{\circ} \mathrm{K}$ too low, compared with standard effective temperature calibrations of (B-V) for giants (cf. e.g., Johnson 1966). If we had taken $T_{e}=4100^{\circ} \mathrm{K}$ rather than $4700^{\circ} \mathrm{K}$, then $\delta T_{e}=-120^{\circ} \mathrm{K}$, and $\Delta(B-V)=0.08$.
} 
2) Planetary nebulae. These are generally thought to descend from stars in the $1-4 M_{\theta}$ range (Miller 1974) and some may therefore belong to the ODP. $\mathrm{He} / \mathrm{H}$ ratios can, of course, be measured directly in the ejected material. Torres-Peimbert and Peimbert (1978), from analysis of 33 planetaries, find $\frac{d \log (\mathrm{He} / \mathrm{H})}{d R}=-0.02$, where $R$ is the distance from the galactic center (in $\mathrm{Kpc}$ ). Measurement of metal abundances (principally 0 ) yield also the correlation $\frac{\Delta Y}{\Delta Z}=2.5$. Barker (1978), on the other hand, finds evidence for only a small inverse gradient of $\mathrm{He} / \mathrm{H}$ with $\mathrm{R}$, and among the 37 planetaries studied by him, there is a spread in $Y$ from 0.14 to 0.40 , with $\langle Y\rangle=0.28$. Since the error in $Y$ is estimated to be only about $\pm 10 \%$, the spread is presumably real, but Barker attributes most of it to inadequate allowance for the incomplete ionization of He. There remain, however, two serious uncertainties: (1) do any disk planetaries in fact descend from stars with masses ${ }_{0}^{5} \mathbb{R}_{0}$ (as required by the values of $\mathrm{Di}_{R G}$ in Table 5), and (2) do the measured $\mathrm{He} / \mathrm{H}$ ratios really reflect the abundances in the primordial stellar object? The existence of a planetary in M 15 (0'Dell, Peimbert, and Kinman 1964) of very low mass (Hawley and Miller 1978) shows that low-mass stars with low $Z$ do indeed pump the planetary nebula domain, but if such objects existed in the metal-rich disk, they might well go unnoticed when compared to the more normal high-mass objects of higher surface brightness. Thus, in connection with the problem of the He-abundance in the old galactic disk, the evidence from planetaries is rather inconclusive. 
3) Old Disk Dwarfs. If He were down in ODD's relative to (say) the Hyades and other young disk dwarfs, we would expect the lower main sequence to be elevated. For example, from Sweigart and Gross (1977), we find that a decrease in $Y$ of 0.07 leads to an increases in $\log L / L_{\theta}$ on the ZAMS of 0.15 , or $\Delta M_{v}=-0.4 \mathrm{mag} .$, for $Z=0.01$. Is there any evidence that this is the case? The distribution of ODD's (defined kinematically as the metamorphs of ODG's) in the $\underline{M}_{b 01}$ vs. $\underline{R}-\underline{I}$ plane shows a scatter in $\underline{M_{b o l}} \sim 0.5$ mag. (Eggen 1973b); most of this is probabiy due to errors in the parallaxes but some might well be due to a range in $Y$ and/or $Z$ amongst ODD's. Of more direct interest here, however, is the possibility that within the group of ODD's, $Y$ is positively correlated with $Z$. If, for example, the ODD's with $Y=0.20$ had $[\mathrm{Fe} / \mathrm{H}]$ typically $\sim-0.4$ whereas $00 \mathrm{D}$ 's with $Y=0.27 \operatorname{had}[\mathrm{Fe} / \mathrm{H}]=+0.1$, then the lowering of the ZAMS owing to the decline in $Z$ would simply be balanced by the raising of the ZAMS owing to a decrease in $Y$ (cf. Sweigart and Gross 1977). The possibility that this remarkable coincidence might actually occur has been noted aTready by Eggen (1973마). Thus our proposed lowering of $Y$ in some ancient disk dwarfs would go essentially unnoticed. Interestingly enough, the correlation would lead to $\frac{\Delta}{\Delta Z} \approx 4$ in the disk, a value somewhat larger than, but probably not incompatible with, that required by the Peimberts to explain the run of $\mathrm{O}$ and $\mathrm{He}$ abundances in planetary nebulae.

On the other hand, if $Y$ and $Z$ were strictly correlated in the disk, then the very nice explanation advanced (Demarque and $\mathrm{McClure} \mathrm{1977a)}$ ) to distinguish the c-m array of 47 Tuc from NGC 2420 would be thwarted. It seems more likely that, as a function of time, there is a general buildup of $Z$ with $Y$, but at any given epoch, there exists considerable scatter in the ratio $Y / Z$. 
I am deeply indebted to the following persons for communicating results in advance of publication: Drs. Lee McDonald, Dave Philip, Dennis Butler, Allan Sandage, Roger Bell, Robert McClure, Allan Sweigart, Peter Gross, Jim Hesser and A. Renzini, whose notes for lectures given at the 1977 Saas-Fee (Switzerland) conference were particularly valuable. Thanks go to Sandy Faber for bringing back the "word" from the 1977 Yale conference on "Stellar Populations in Galaxies" and to Nick Suntzeff for good discussions and help surveying the literature. Special appreciation is recorded here to Ed Langer whose stimulating ideas and cheerful intelligence helped bring the author through an especially trying period of scientific isolation and general ennui.

This research was supported by NSF Grant AST 74-13717.

\section{References}

Arp. H. 1965 in "Stars and Stellar Systems", Vol. V (ed. A. Blaauw and M. Schmidt), (Chicago: Univ. of Chicago Press), p. 401.

Arp, H. and Hartwick, D. 1971, Ap.J., 167, 499.

Barker, T. 1978, Ap.J., (in press).

Be11, R., Eriksson, K., Gustafsson, B., and Nordlund, A. 1976, A.A. Supplements, 23,37 .

Butler, D. 1975a, Ap.J., 200, 68.

Butler, D. 1975b, Pub. A.S.P., 87, 559.

Butler, D., Be11, R., Dickens, R., and Epps, E., 1978 (preprint).

Carbon, D., Langer, G. E., Butler, D., Kraft, R., Nocar, J., Kemper, E. 1978 (in preparation).

Christy, R. 1966, Ap.J., 144, 108. 
Cohen, J. 1975, Bull. A.A.S., I, 446.

Demarque, P., and McClure, R. 1977a, Ap.J., 213, 716.

Demarque, P., and McClure, R. 1977b, in Yale Conference on "Stellar Populations in Galaxies", p. 199.

Dickens, R. 1970, Ap.J. Suppl., 22, 249.

Eggen, O. 1973a, Pub. A.S.P., 85, 542.

Eggen, 0. 1973b, Ap.J., 182, 821.

Faulkner, J. 1966, Ap.J., 144, 978.

Fusi-Pecci, F., and Renzini, A. 1975, Astron. \& Ap., 39, 413.

Gross, P. 1973, M.N.R.A.S., 164, 65.

Hartwick, D. 1968, Ap.J., 154, 475.

Hartwick, D., and Hesser, J. 1974, Ap.J. Letters, 197, L129.

Hawley, S., and Miller, J. S. 1978, Ap.J., (in press).

Hemenway, M. 1975, A.J., 80, 199.

Hesser, J., Hartwick, D., McClure, R. 1976, Ap.J. Letters, 207, L113.

Iben, I. 1971, Pub. A.S.P., 83, 697.

Iben, I. 1974, Ann. Rev. Astron. \& Ap., 12, 215.

Johnson, H. L. 1966, Ann. Rev. Astron. \& Ap., 4, 193.

Kinman, T. 1959, M.N.R.A.S., 119, 157, 538 and 559.

Langer, G. E. 1977, Ap.J. Letters, (in press).

McClure, R., Forrester, W., and Gibson, J. 1974, Ap.J., 189, 409.

McClure, R., and Osborn, W. 1974, Ap.J., 189, 405.

McDonald, L. 1976, Thesis, Univ. of Calif., Santa Cruz.

Miller, J. S. 1974, Ann. Rev. Astron. \& Ap., 12, 331.

Nisson, P. 1976, in I.A.U. Symposium No. 72, (ed. B. Hauck and P. Keenan), (Dordrecht: Reidel Pub. Co.), p. 81.

O'Del1, C., Peimbert, M., and Kinman, T. D. 1964, Ap.J., 140, 119.

Oosterhoff, P. 1939, Observatory, 62, 104. 
Philip, A. G. D. 1977, private communication.

Preston, G. 1959, Ap.J., 130, 507.

Preston, G. 1961, Ap.J., 134, 633 and 651.

Renzini, A. 1977, Lectures given at the 7th Advanced Course Saas-Fee (Switzerland), March 28 - April 2, on "Advanced Stages in Stellar Evolution".

Rood, R. 1972, Ap.J., 177, 681.

Rosino, L. 1966, Ap.J., 144, 903.

Sandage, A. 1969, Ap.J., 157, 515.

Sandage, A. 1970, Ap.J., 162, 841.

Sandage, A., and Eggen, 0. 1969, Ap.J., 158, 685.

Sandage, A., and Hartwick, D. 1977, A.J., 82, 459.

Sandage, A., and Katem, B. 1964, Ap.J., 139, 1088.

Sandage, A., and Smith, L. 1966, Ap.J., 144, 886.

Sandage, A., Smith, L., and Norton, R. 1966, Ap.J., 144, 894.

Stellinwerf, R. 1975, Ap.J., 195, 441.

Stepien, K. 1972, Acta Astronomica, 22, 175.

Sturch, C. 1967, Ap.J., 148, 477.

Sweigart, A., and Gross, P. 1976, Ap.J. Supp1., 32, 367.

Sweigart, A., and Gross, P. 1977, preprint.

Taam, R., Kraft, R., and Suntzeff, N. 1976, Ap.J., 207, 201.

Tinsley, 8., and Gunn, J. 1976, Ap.J., 206, 525.

Torres-Peimbert, S., and Peimbert, M. 1978, preprint.

van Albada, T., and Baker, N. 1973, Ap.J., 185, 477.

van den Bergh, S. 1969, Ap.J. Supp1., 19, 145.

Wilson, 0. 1976, Ap.J., 205, 823.

Woolley, R., Harding, G., Cassells, A., and Saunders, J. 1965, Royal Obs. Bul1., No. 97. 
D I S C US I O N of paper by KRAFT:

REIMERS: Did I understand you correctly in that you no longer require exceptionally high mass-loss for the progenttors of metal-rich field RR Lyraes?

KRAFT: No, one still requires a total mass-loss, using the FusiPecci and Renzini theory, of between 0.36 and $0.28 \mathrm{M}$, if the age of the old disk is about 11 billion years $\left(\mathrm{Table}^{\Theta_{5}}\right)$; if the age is older, say about 14 billion years, then the requirement on $\Delta \mathrm{M}$ is smaller, and for $\mathrm{Y}=0.27$, you can just jump the $R R$ Lyrae domain, as required by the metal-rich cluster NGC 6712 . But metal-rich field stars at $Y=0.20$ require a little more mass-loss since the turn-off mass is higher at the same age. Thus still only a few ODG's with excessive mass-loss can metamorphose into MR-RR's - luckily, otherwise the sky would be filled with MR-RR's!

WEYMANN: Is there any evidence (aside from Cohen's work) that the mass-loss required to produce RR $L_{Y r}$ stars takes place continuously, as opposed to discrete events like planetary nebulae?

KRAFT: None that I know of. I think most people believe, though, the planetary ejection is a post-HB event, and terminates the second ascent of the red giant branch, maybe resulting from an "ultimate" helium shell fiash. But, of course, all these things are somewhat problematical.

KOPAL: The (one) eclipsing system which is a member of $w$ Cen appears to be unevolved (i.e., is of the detached type, not semi-detached). Which helium contents would be compatible with that fact?

KRAFT: I do not know. As you say, the result is quite odd; globular clusters do sometimes seem to contain objects that "defy" explanation by the normal ideas of SET.

PEL: I would like to draw attention to a recent Ph.D. thesis at Leiden University by $\mathrm{Dr}$. J. Lub, who has made a very extensive photometric study of RR Lyrae stars in the solar neighbourhood (in the Walraven VBLUW system). Unfortunately, I cannot quote figures, but Dr. Lub's analysis is relevant to several of the problems just discussed by Dr. Kraft.

KRAFT: Thank you for the information. I shall look forward to seeing Dr. Lub's results, and would appreciate having a preprint.

GEYER: For historical reasons, I like to mention that before Preston's 1959 paper, the metal content of the RR Lyrae field stars was pointed by Parenago in Moskau, Dranowska at Torun and Notni in Babelsberg. Concerning the different RR Lyrae groups within one globular cluster this was for the first time shown by Szeidl in Budapest for M3 in 1965 and by Szeidl and myself for w Cen $(1965,1970)$. My question is now, how you could explain such an effect by a Y-content reduction for the two groups and reconcile this enigma for the origin of the globular cluster stars?

KRAFT: MY treatment does not require a reduction in $\mathrm{Y}$ for globular cluster stars: these would still have $Y$ about 0.27 . I suggest that $Y$ about 0.20 would explain the periods and luminosities of the MR-RR's of the Galactic disk only (i.e., those with 
$\Delta S=0,1,2)$. So $f a r$ as I can see, the two metal groups in $w$ cen would probably have the same high value of $Y$.

RENZINI: Concerning the problem of the missing neutral hydrogen in globular cluster: first, one does not expect that hydrogen is neutral as i) the velocity of the red giant winds (about 20 $\mathrm{km} / \mathrm{s}$ ) is large enough that hydrogen is ionized when two winds collide, and second, each evolving star in a globular cluster spends some $10^{4}$ years as a hot UV-star before dying as a white dwarf providing enough UV-photons to largely ionize about $0.3 M_{\theta}$ of hydrogen which have been lost in the previous evolutionary phases.

Freeman et al., Ap. J., 1977, have computed models of the gas distribution in globular clusters, showing that in most cases the gas is lost by the cluster at the same rate at which it is produced by red giants. The amount of gas stationary present in one cluster is typically about $1 M_{0}$ in the form of ionized hydrogen.

Concerning the problem of mass-loss in population II star, I would like to stress that the observed trend of the horizontal branch morphology with metal abundance sets quite strict constraints to the dependence of the mass loss rate on metal abundance $(z)$. Assuming $M$ proportional to $z \alpha$ we find that $\alpha$ must be less than about 0.2 , otherwise the trend of the HB morphology would be just opposite of that which is observed. In other words, the metal abundance seems to play a minor role in determining the mass-loss rate of red giants. 